\title{
Synchronized switch harvesting applied to piezoelectric flags
}

\author{
Miguel Piñeirua ${ }^{1}$, Sébastien Michelin $^{2}$, Dejan Vasic $^{3}$ and Olivier Doaré ${ }^{1}$ \\ ${ }^{1}$ Unité de Mécanique, ENSTA Paristech, Palaiseau, France \\ ${ }^{2}$ LadHyX-Dpartement de Mcanique, CNRS-Ecole Polytechnique, F-91128 Palaiseau, France \\ ${ }^{3}$ SATIE, ENS Cachan, Cachan, France
}

Received 8 January 2016, revised 13 May 2016

Accepted for publication 31 May 2016

Published DD MM 2016

\begin{abstract}
In this article the energy transfer between a flow and a fluttering piezoelectric plate is investigated. In particular, the benefits of the use of a synchronized switch harvesting on inductor (SSHI) circuit are studied. Both wind tunnel experiments and numerical simulations are conducted in order to analyze the influence of the switching process on the dynamics and the efficiency of the system. Numerical simulations consist of a weakly nonlinear model of a plate in axial flow equipped with a single pair of piezoelectric patches, discretized using a Galerkin method where basis functions are the modes of the plate in vacuum. The discretized model is then integrated in time. The results presented in this paper show that a significant improvement of the harvested energy can be obtained using SSHI circuits compared to basic resistive circuits. It is also shown that for strongly coupled systems, the switching process inherent to he SSHI circuit has a significant impact on the dynamics of the flag, which tends to decrease the relative efficiency gain.
\end{abstract}

Keywords: piezoelectricity, fluid-structure interactions, energy harvesting, active circuits

(Some figures may appear in colour only in the online journal)

\section{Introduction}

In recent years, piezoelectric materials have received considerable attention in order to convert mechanical energy associated with ambient vibrations into electrical form, and power mobile and wireless microsystems [1]. Piezoelectric materials are also interesting to design new kinds of electric transformers [2]. An important ingredient is however the existence of a vibration source. Hence, flow-induced vibrations have recently been considered as potential routes to generate spontaneous and self-sustained vibrations of solid structures that can then be used to power an electric generator (piezoelectric or other): these include vortex-induced vibrations [3], coupled mode flutter [4], galloping [5], or flutter instability of flags [6].

The latter is the focus of the present article: a plate covered by piezoelectric patches and placed in an axial air- or water-flow of sufficient velocity undergoes large-amplitude self-sustained oscillations above a critical velocity threshold. These oscillations originate from an instability of the equilibrium position, resulting from the interaction of the flow forces with the solids inertia and rigidity. This instability is referred to as flag coupled mode flutter and has been the subject of numerous research papers in the last decades [7, 8]. The idea of using fluttering piezoelectric plates to harvest flow kinetic energy was born in the early 2000s [9] and numerous studies followed. Recently the influence of piezoelectric coupling on the stability properties and dynamics of the plate has been characterized $[10,11]$, as well as the importance of mechanical-electrical synchronization [12, 13] and of piezelectric patches' positions and dimensions [14] in order to maximize the efficiency of the system.

Electro-mechanical couplings of common piezoelectric materials are relatively low and an important challenge lies in the optimization of the output circuit to maximize the energy transfer. A broadly used technique in the optimization of mechanical to electrical power conversion from vibrating structures is the synchronized switch harvesting on inductor (SSHI) [15], which aims to synchronize the voltage and current in the output circuit so as to maximize the energy transfer. There are two circuit topologies of SSHI: series and parallel. In the latter, the circuit includes an active switch that briefly connects a parallel inductive branch when the displacement of the mechanical system is maximal, in order to 


\section{(a)}

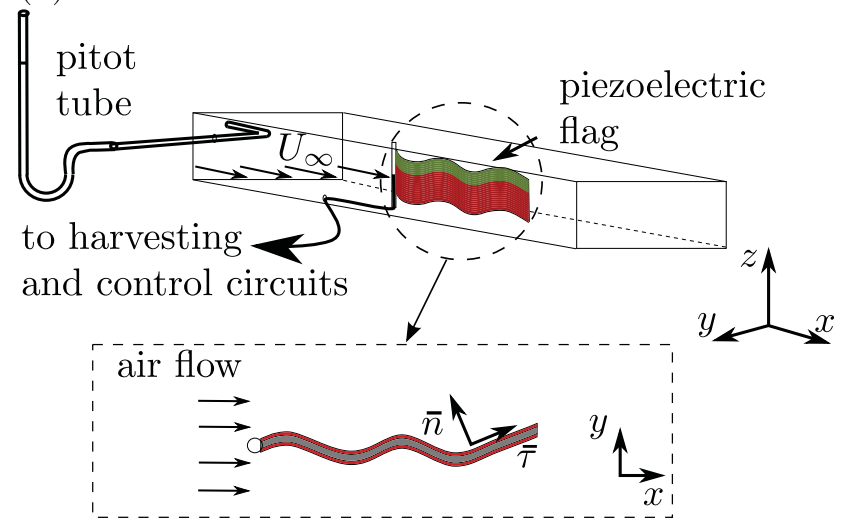

(c)

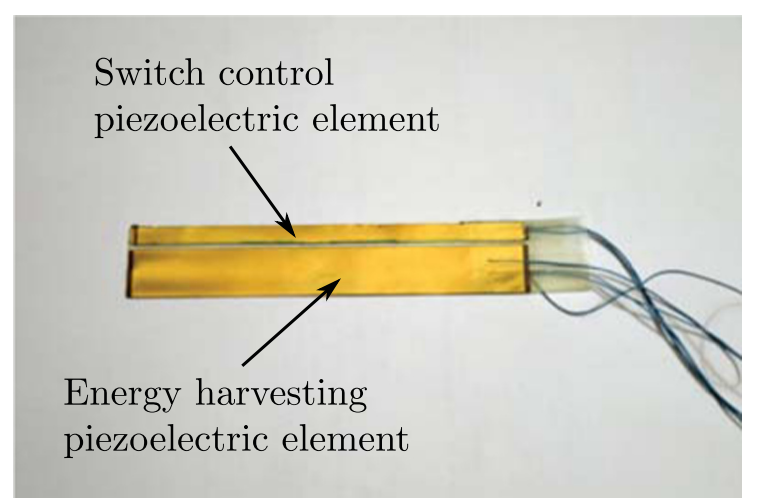

(b)

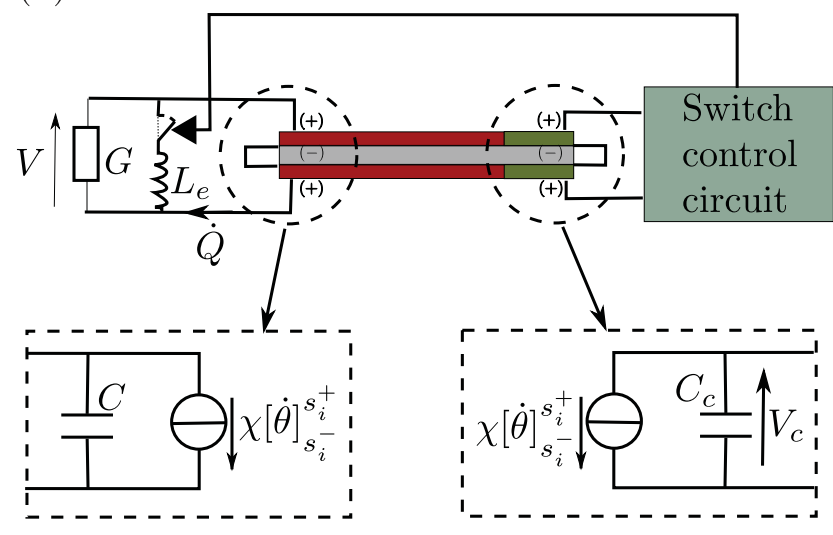

(d)

Figure 1. (a) Description of the experimental setup. (b) Connection diagram of the experimental setup. The energy harvesting and switch control piezoelectric elements are shown in red and green respectively. (c) Schematic of the multilayer composition of the piezoelectric flag. (d) Temporal evolution of $v$ and $\theta$ for standard and SSHI configurations in open circuit conditions.

reverse the voltage applied on the output load. If the system is properly tuned, this synchronization technique can significantly increase the electrical power that can be extracted from the vibrations of the mechanical system [15]. The SSHI technique is widely studied in the domain of power electronics and various improvements have recently been proposed, such as self-powered SSHI $[16,17]$.

The SSHI technique is generally applied in the optimization of the energy harnessing from vibrating piezoelectric structures excited by external driving forces. Nevertheless, the application of this technique to the flow-induced vibration of structures and its impact on the energy conversion efficiency of these kind of systems has not been studied yet. Indeed, in the case of fluttering piezoelectric plates, the coupling between the fluid-structure system and the external circuit is of great importance in the overall efficiency of the system, as the electric loading can dramatically influence the plate dynamics.

Based on experiments and numerical simulations, the objective of the present work is to characterize the influence of the SSHI parallel technique on the coupled fluid-solidelectric dynamics of a piezoelectric fluttering flag, and to quantify the improvement on the energy harvesting efficiency it can provide. The paper is organized as follows: in section 2, the piezoelectric flag system and SSHI circuits are presented and modeled, together with the experimental setup and numerical simulations techniques. In section 3 , the experimental and numerical data are presented for both weak and strong couplings of the mechanical and electrical systems, and the influence of the circuit's design on the harvesting efficiency is analyzed. Finally, section 4 summarizes our results and offers some perspectives.

\section{Piezoelectric flag: modeling and experiments}

In this section we present a series of numerical simulations and experiments aiming to study the impact of the synchronized switching on the energy harvesting efficiency of a piezoelectric flag. We focus here on a simple configuration consisting of a flexible piezoelectric plate immersed in an axial wind flow (figure 1(a)). The piezoelectric plate is connected to an electric circuit which consists of a resistive charge with a parallel inductive branch that can be switched on/off in order to inverse the piezoelectric voltage. A physical model of the system used in the numerical simulations is presented in the next section 2.1. Afterwards, a detailed 
description of the experimental setup and materials are reported in section 2.4 .

\subsection{Physical model}

The piezoelectric flag considered here consists of an inextensible thin flexible plate of length $L$ and width $H$. The plate is clamped at its leading edge and placed in an axial wind flow of density $\rho$ and velocity $U_{\infty}$ (figure $1(\mathrm{a})$ ). The entire plate is covered by two pairs of piezoelectric patches, and within each pair, the negative electrodes of the piezoelectric patches are shunted through the flag. The lineic mass and bending rigidity of the assembly are $\mu$ and $B$, respectively.

The output circuit is connected to the positive electrodes of the first piezoelectric pair, referred to in the following as harvesting assembly (see figure 1(b)). In the present approach, the energy dissipated in the resistance models the harvested energy. The positive electrodes of the second pair, referred to as the controlling patch assembly, are connected to the switch control circuit used to detect maxima of the plate's deflection by means of zero crossing velocity detection.

Beyond a critical wind velocity, the plate starts to flap in a self sustained oscillatory regime. In the following, we consider only the two-dimensional motions of the plate in the $(x, y)$ plane. The deformation of the piezoelectric patches induces a charge displacement between their electrodes and a net current within the resistive load. The total charge displacement $Q$ in the harvesting circuit is related to the plate's deformation and voltage $V$ by [10],

$$
Q=\chi \theta(L)+C V,
$$

where $\theta(L)$ is the local orientation of the plate at the trailing edge with respect to the flow direction. $C$ and $\chi$ are the capacitance and electromechanical coupling coefficients of the harvesting piezoelectric assembly, respectively [18]. The voltage at the electrodes of the harvesting assembly induces a feedback mechanical forcing due to the inverse piezoelectric effects, in the form of an additional torque on the flag. Assuming that the flag itself follows an inextensible EulerBernoulli beam equation, its dynamics are described by

$$
\mu \ddot{\mathbf{X}}(S, t)=\left[\mathcal{T} \boldsymbol{\tau}-M^{\prime} \mathbf{n}\right]^{\prime}+\mathbf{f}_{\text {fluid }}, \quad \mathbf{X}^{\prime}(S, t)=\boldsymbol{\tau},
$$

where the total torque within the flag is given by

$$
M=B \theta^{\prime}-\chi V F_{v} .
$$

In equation $(2),(\boldsymbol{\tau}, \mathbf{n})$ are the unit tangent and normal vectors to the flag, $f_{\text {fluid }}$ is the loading exerted by the surrounding fluid and $\mathcal{T}$ the tension within the flag. $F_{v}$ is the polarization function of the piezoelectric element. In the present approach, $F_{v}(S)=\mathcal{H}(S)-\mathcal{H}(S-L)$, with $\mathcal{H}$ the Heaviside step function. Derivation with respect to the curvilinear coordinate $(S)$ and time $(t)$ are respectively noted ()$^{\prime}$ and $(\dot{)}$. For slender flags, the fluid loading $\mathbf{f}_{\text {fluid }}$ is modeled locally in terms of the relative velocity of the flag to the background flow, using the large amplitude elongated body theory [19, 20] combined with a resistive drag accounting for lateral flow separation $[12,21,22]$. The feedback of the controlling patch assembly is neglected: the second pair of piezoelectric patches act only as a sensor that monitors the deformation of the plate.

The harvesting circuit consists of a purely resistive load connected in parallel to an inductance $L_{e}$ associated with a switching device controlled by the second piezoelectric pair. The harvested energy is modeled here as the energy dissipated in the resistive output. When the switching device is open (figure 1(b)), the current through the inductance $L_{e}$ is zero, and $Q$ and $V$ are simply related through Ohm's law:

$$
\dot{Q}+G V=0,
$$

where $G$ is the conductivity of the load. When the switching device is closed, Kirchhoff's law becomes

$$
\ddot{Q}+G \dot{V}+\frac{V}{L_{e}}=0
$$

due to the presence of the inductance.

Using $L, L / U_{\infty}, U_{\infty} \sqrt{\mu L / C}$ and $U_{\infty} \sqrt{\mu C L}$ as characteristic length, time, voltage and charge respectively, the coupled dynamics of the flag and harvesting device can be completely described by five non-dimensional parameters, namely

$$
\begin{gathered}
U^{*}=L U_{\infty} \sqrt{\frac{\mu}{B}}, \quad M^{*}=\frac{\rho H L}{\mu}, \\
\alpha=\chi \sqrt{\frac{L}{B C}}, \quad \beta=\frac{U_{\infty} C}{L G}, \quad \Gamma=\frac{L}{U_{\infty} \sqrt{L_{e} C}} .
\end{gathered}
$$

The first four coefficients are the dimensionless flow velocity, fluid-solid mass ratio, electro-mechanical coupling factor, and tuning coefficient of the fluid-solid and electric systems respectively. The last coefficient, $\Gamma$, is a relative measure of the fundamental frequency of the $L_{e} C$ circuit when the switch is closed with respect to the characteristic time scale of the flag dynamics. In the following, the $L_{e} C$ reversal time scale will be much shorter than the flapping period and $\Gamma \gg 1$.

In the present study the fluid-structure parameters will be fixed for the particular case of a flexible plate in air at constant speed $U_{\infty}$ just above the instability threshold $\left(U^{*}=14.5\right.$, $\left.M^{*}=0.67\right)$. A particular attention will be dedicated to the coupling factor $\alpha$ and tuning coefficient $\beta$, since they are indeed relevant in the evaluation of the active circuit system presented in this work.

\subsection{Standard and SSHI energy harvesting}

In the following, two different energy harvesting methods are compared. The standard technique considers a purely resistive load without any inductance (i.e. the switch is not operated). In the SSHI technique, the inductive branch is closed when $\theta(L)$ reaches its maximal and minimal values (see figure 1(b)). When the inductive branch is closed, the inductance, capacitance and the resistance form a resonant circuit of short oscillating period $T \sim \sqrt{L_{e} C}$.

Following [15], the switch is re-opened after one half oscillation period of the $L_{e} C$ circuit resulting in an inversion of the voltage across the resistive load. In order to achieve adequate shifting, the oscillating period of the $L_{e} C$ circuit must be small compared to the oscillation period of the flapping flag, hence $\Gamma \gg 1$. Typically, some losses occur 
during the voltage inversion process which are related to energy dissipation in the coil. These losses are taken into account through an electric quality factor $Q_{i}$, such that the voltage after the short inverting process at $t=t_{s}$ is obtained as:

$$
V\left(t_{s}^{+}\right)=-V\left(t_{s}^{-}\right) \mathrm{e}^{\frac{-\pi}{2 Q_{i}}}
$$

The impact of $Q_{i}$ on the energy harvesting efficiency will be discussed in the following.

In both standard and SSHI harvesting techniques, the power dissipated in the resistive load is computed as the temporal average $P_{e}=\left\langle V^{2} G\right\rangle$ over one oscillation period. The efficiency of the system, $\eta=P_{e} / P_{f}$, is defined as the ratio of the harvested electric power $P_{e}$ to the fluid kinetic energy flux $P_{f}$ through the vertical cross-section occupied by the flag $P_{f}=1 / 2 \rho A H U_{\infty}^{3}$, where $A$ is the oscillation amplitude of the trailing edge of the plate.

All other parameters being fixed, the harvesting efficiency vanishes in the open circuit $(G=0)$ and short circuit $(G=\infty)$ limits since $P_{e} \rightarrow 0$ in both cases. Therefore, $\eta$ is maximum for an intermediate value of $G$ (or $\beta$ ). In the following, $\beta$ is therefore varied to find the maximum dissipated power, which corresponds to a tuning of the flapping frequency to the characteristic timescale of the RC circuit consisting of the piezoelectric capacitance and output resistor [12].

\subsection{Numerical solution}

The non-dimensional form of the dynamical equations for the plate and circuit are solved in the weakly nonlinear limit following the approach described in details in [14]. Equation (2) is first projected onto the $x$ - and $y$-directions and the horizontal projection is used to eliminate the tension force from the $y$ projection using the inextensibility condition. Retaining only nonlinear terms up to $\mathcal{O}\left(y^{3}\right)$, a classical Galerkin decomposition is then employed: expanding the $y$ displacement on clamped-free beam eigenmodes, the beam equation is projected on the same set of eigenmodes. After truncation to $N$ linear modes, the resulting coupled system of equations are integrated numerically using an explicit semiadaptive fourth order Runge-Kutta method.

\subsection{Experimental setup}

The piezoelectric plate's center core consists of a doublesided adhesive tape of length $L=10 \mathrm{~cm}$, width $H=3 \mathrm{~cm}$ and thickness $h_{s}=100 \mu \mathrm{m}$. Both sides of the tape are totally covered by a piezoelectric PVDF film of length $L$ and width $0.75 \mathrm{H}$, for the energy harvesting piezoelectric patch and $0.25 \mathrm{H}$ for the controlling piezoelectric patch. In both cases the negative polarity of the piezoelectric film faces inwards. The thickness of the piezoelectric film is $50 \mu \mathrm{m}$. Figure 1(c) shows a schematic view of the plate's structure. The piezoelectric PVDF films (Piezotech ${ }^{\circledR}$ ) are covered with an external $\mathrm{Cr} / \mathrm{Au}$ layer which serves as the electrodes, connecting the piezoelectric patches to the energy harvesting and switching
Table 1. Piezoelectric element characteristics.

\begin{tabular}{lcc}
\hline & Harvesting element & Zero detection element \\
\hline Length $(\mathrm{cm})$ & 10 & 10 \\
Width $(\mathrm{cm})$ & 2.25 & 0.75 \\
Thickness $(\mu \mathrm{m})$ & 50 & 50 \\
\hline
\end{tabular}

Table 2. Measured experimental parameters.

\begin{tabular}{|c|c|c|}
\hline Symbol & Description & Value (unit) \\
\hline \multicolumn{3}{|c|}{ Fluid/structure parameters } \\
\hline$\mu$ & $\begin{array}{l}\text { Mass per unit length of } \\
\text { the piezoelectric flag }\end{array}$ & $7 \times 10^{-3}\left(\mathrm{~kg} \mathrm{~m}^{-1}\right)$ \\
\hline$U_{\infty}$ & Windspeed & $12.7\left(\mathrm{~m} \mathrm{~s}^{-1}\right)$ \\
\hline$L$ & Flag length & $10(\mathrm{~cm})$ \\
\hline$H$ & Flag width & $3(\mathrm{~cm})$ \\
\hline$B$ & $\begin{array}{l}\text { Bending rigidity of } \\
\text { the flag } \\
\text { (including piezoelectric } \\
\text { elements) }\end{array}$ & $5.3 \times 10^{-5}\left(\mathrm{Nm}^{2}\right)$ \\
\hline \multicolumn{3}{|c|}{ Electric parameters } \\
\hline C & Circuit capacitance & $58(\mathrm{nF})$ \\
\hline$L_{e}$ & $\begin{array}{l}\text { Switching branch } \\
\text { inductance }\end{array}$ & $50(\mathrm{mH})$ \\
\hline$Q_{i}$ & Electric quality factor & 1.9 \\
\hline$G$ & $\begin{array}{l}\text { Load conductivity } \\
\text { (variable) }\end{array}$ & $1 \times 10^{-6}-1 \times 10^{-4}(1 / \Omega)$ \\
\hline$\chi$ & $\begin{array}{l}\text { Electro-mechanical cou- } \\
\text { pling coefficient }\end{array}$ & $3.6 \times 10^{-7} \pm 3 \times 10^{-8}(\mathrm{C})$ \\
\hline
\end{tabular}

control circuits. The piezoelectric element characteristics are summarized in table 1 .

The piezoelectric plate is placed in a wind tunnel of $10 \times 5 \mathrm{~cm}$ section with transparent walls allowing for a visual access [23]. The overall piezoelectric coefficient $\alpha$ of the system is determined experimentally by measuring the generated output voltage corresponding to the real time plate deformation recovered via high speed camera acquisition. For our system we obtain a value of $\alpha=0.065 \pm 0.007$ [24].

The harvesting piezoelectric patch is connected to a purely resistive circuit of variable conductivity. The voltage across the resistive load is used to determine the dissipated power $P_{e}$. The controlling piezoelectric patch is connected to a high-impedance electronic circuit (i.e. the electric charge displacement in that second piezoelectric pair is negligible). When the voltage (or equivalently the trailing edge's deflection, equation (4)) reaches a maximum or a minimum value, the control circuit generates a pulse that closes the inductive branch in the harvesting circuit. The reopening of the switch is controlled manually by adjusting the triggering threshold in order to get only one half oscillating period of the $L_{e} C$ circuit.

The inductive branch consists of a $L_{e}=50 \mathrm{mH}$ inductor, which along with the piezoelectric capacitance $C=58 \mathrm{nF}$, give $\Gamma \sim 150$. In this way we assure that the oscillation period of the $L_{e} C$ circuit is much smaller than the flapping period of the flag. The factor $Q_{i}$ related to dissipative losses in the inductor is measured directly from the experiments, by 


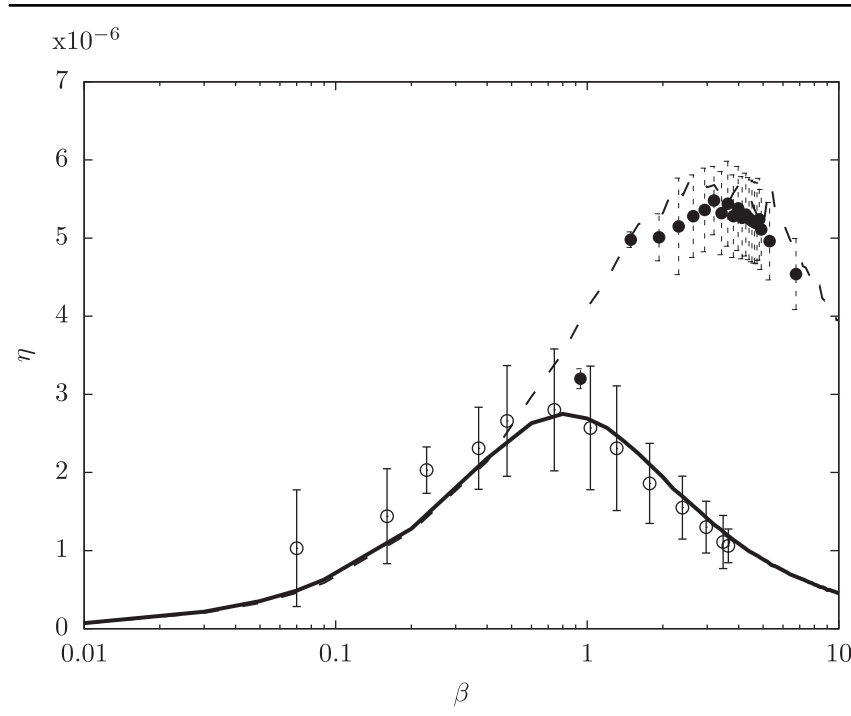

Figure 2. Harvested energy efficiency as a function of the tuning parameter $\beta$ obtained in experimentally for the standard (open circles) and SSHI (filled circles) techniques, for $\alpha=0.065, Q_{i}=1.9$ and $\Gamma=180$. The corresponding results of numerical simulations are shown as solid and dashed lines.

comparing the voltage values before and after the inversion. In our experiments we obtain $Q_{i} \sim 1.9$, which is also chosen for consistency in the numerical simulations.

\section{Results}

Both standard and SSHI harvesting techniques are now compared using a combination of numerical simulations and experimental measurements. In section 3.1, the results of numerical simulations are compared to experiments in the case of a weakly coupled system $\alpha \ll 1$. Section 3.2 then presents numerical simulation results for a strongly coupled system $(\alpha=0.5)$ and analyzes the impact of the coupling factor on the harvesting efficiency and the plate dynamics for a strongly coupled system.

\subsection{Weakly coupled systems}

In figure 2 we present the results of our experiments and numerical simulations comparing the standard and SSHI techniques.

As reported in previous works [15] for other types of vibrating systems, the SSHI harvesting technique substantially improves the harvesting efficiency of the system in the case of a weakly coupled system (figure 2). An increase of the harvesting efficiency of about $100 \%$ is observed in comparison with the standard harvesting technique. Also, the maximum efficiency is shifted to higher $\beta$ values with respect to the standard case.

The experimental efficiency of the SSHI technique is however limited by the low quality factor value of the inductive circuit $Q_{i} \sim 1$.9. In previous works on SSHI circuits $[15,17]$, the harvested power can be incremented up to $4-5$ times with higher quality factors in the switching processes $\left(Q_{i} \sim 5\right)$.

Due to the limitations of the electromechanical conversion factor of the PVDF film used in our experiments (which corresponds to a value of $\alpha=0.065$ ), our results remain in a weakly coupled regime where the dynamics of the piezoelectric flag stay unperturbed by the electrical circuit charge. Nevertheless, based on the good agreement between our experimental results and our numerical simulations, a numerical study of the impact of high coupling factors on the harvesting efficiency of the system is presented in the following section.

\subsection{Strongly coupled systems}

Large values of the electromechanical coupling factor generate larger charge displacements within the circuit for a given deformation, and thus enhance energy harvesting. For strongly coupled systems, the feedback piezoelectric torque on the flag also has an important impact on the fluid-solidelectric dynamics of the system, which has been showed to substantially affect the overall efficiency of the system [13]. Moreover, in the case of a strongly coupled SSHI device, the sudden changes in the voltage polarity during the switching process can considerably perturb the plate dynamics.

This impact is illustrated on figure 3: for weak coupling, the dynamics of the plate remain unchanged between the standard and SSHI configurations (figure 3(a)). However, for strong coupling, the SSHI configuration significantly modifies the dynamics with a reduction of the flapping amplitude and the introduction of high-frequency components in the plate's vibrations near the switching event. These modifications of the dynamics also impact the efficiency. This is illustrated here on figures 3(b) and (d) showing the recovered energy cycle for the same weakly and strongly coupled systems. Over one cycle, the energy transferred to the output circuit is equal to the enclosed area within the $\theta-v$ trajectory. As expected, for strongly coupled systems the amount of harvested energy per cycle is significantly larger than that of a weakly coupled system. However, the relative output power increase between the standard and SSHI configurations is smaller than for weakly coupled systems. In other words, for larger electro-mechanical coupling, the SSHI advantages are reduced (see figure $4(\mathrm{~b})$ ).

The core idea of SSHI is to synchronize the circuit's dynamics to the plate's motion to ensure a maximum transfer of energy to the electrical system. This ensures a maximum extraction of the mechanical energy of the flag, thereby reducing its flapping amplitude and the amount of mechanical energy available for conversion. The perturbation of the plate's dynamics due to the sudden switching of the inductive branch in the harvesting circuit (as shown in figure 3) could also explain the reduction of the SSHI advantages in strongly coupled systems.

The positioning of the piezoelectric elements on the flag also plays an important role, as it has been shown in previous works [14]. In figure 4(a) we compare the efficiencies of a fully covered piezoelectric flag to those of a flag with an 
(a)

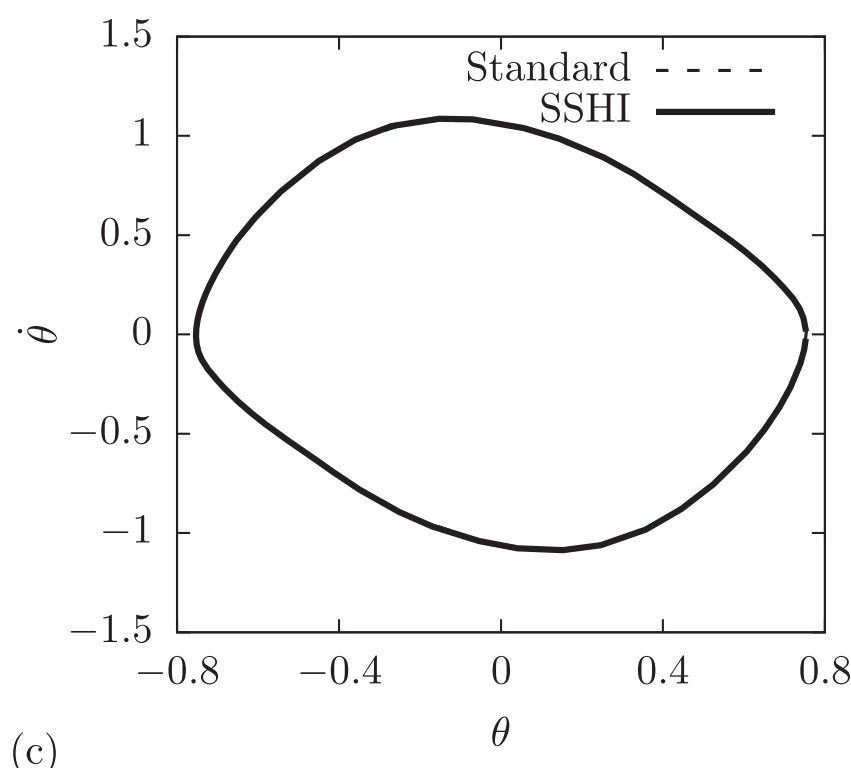

(c)

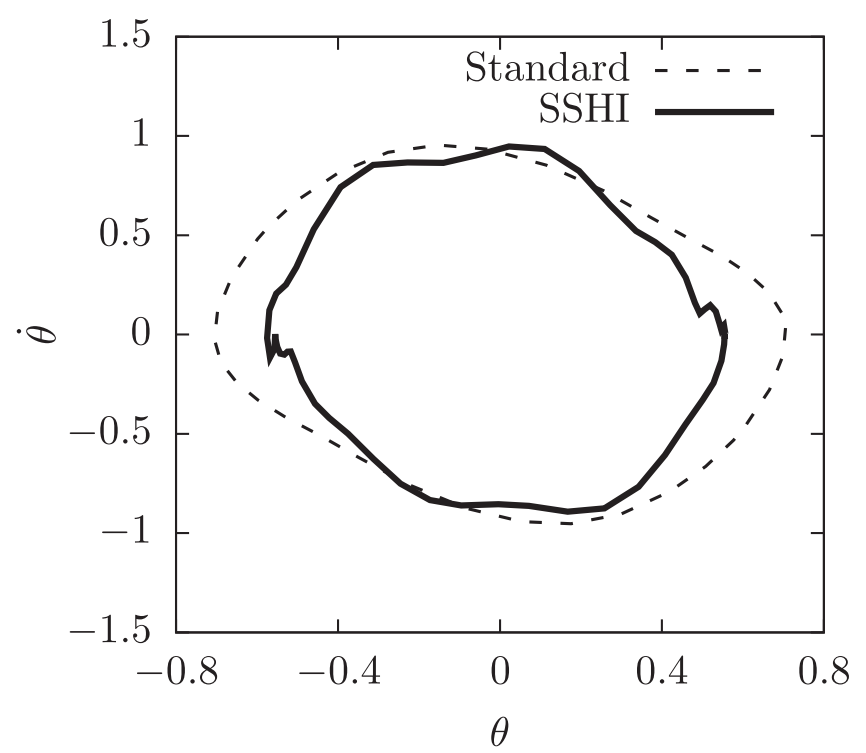

(b)

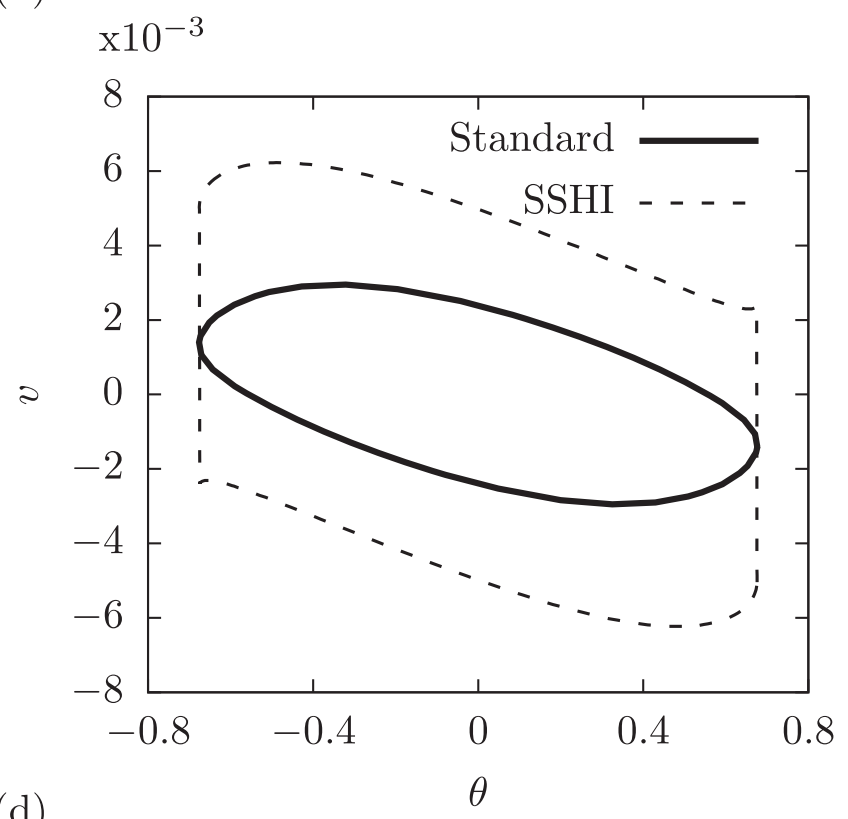

(d)

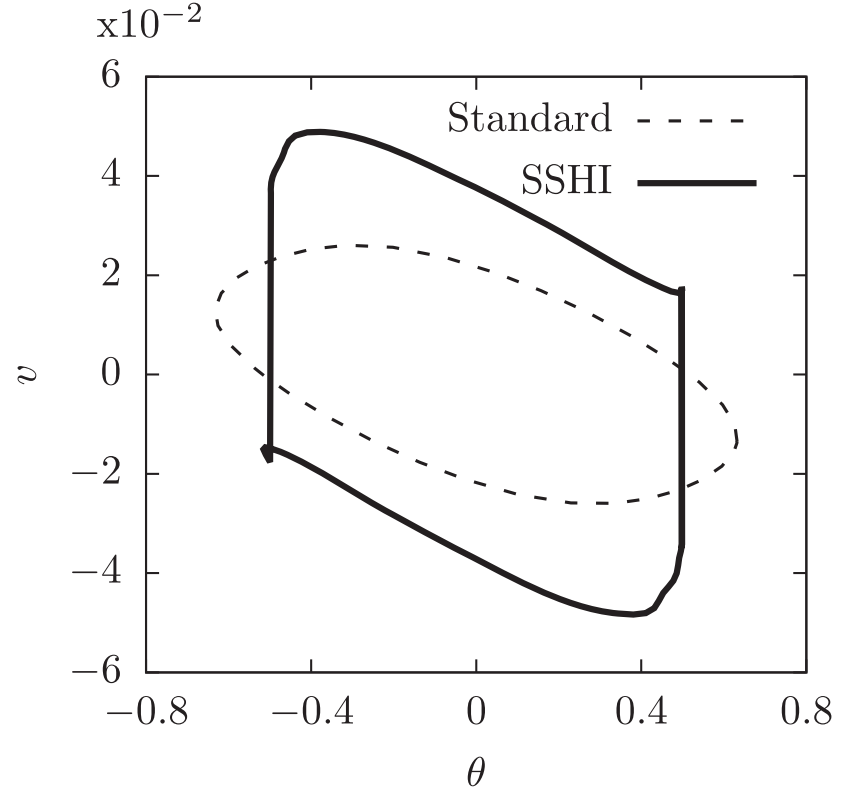

Figure 3. Phase-space trajectory for the trailing edge orientation (left) and non-dimensional output voltage cycle (right) for $\alpha=0.05$ (top) and $\alpha=0.5$ (bottom), and $Q_{i}=1.9$ and $\Gamma=180 . \beta$ is chosen to achieve a perfect tuning and maximum energy transfer: $\beta=0.9$ (std) and $\beta=4$ (SSHI).

optimized piezoelectric element distribution. We observe that the overall efficiency of the system is improved by an optimal positioning of the piezoelectric element for both standard and SSHI harvesting. Also, the optimal positioning of the piezoelectric elements improves the SSHI configuration effectiveness with respect to the standard configuration in about $10 \%$ (figure 4(b)).

Finally, we investigate the importance of the electric quality factor $Q_{i}$ in the efficiency of the SSHI technique. Confirming the results obtained in previous studies [15, 17], the harvested power substantially increases with the quality factor (figure 5). We observe that the tuning parameter $\beta$ maximizing the efficiency also increases with $Q_{i}$ : the maximum efficiency is obtained for larger output resistances. In standard energy harvesting configurations one generally obtains optimal $\beta$ values near unity $[12,14]$. Indeed, in such simple electrical configurations, power production is maximized when the characteristic timescales of the mechanical system $(L / U)$ and of the output circuit $(C / G)$ are comparable. However, in the SSHI case the synchronization between the mechanical and electrical systems is artificially forced, hence, the sense of $\beta$ as a measure of mechanical/electrical synchronization is lost. 
(a)

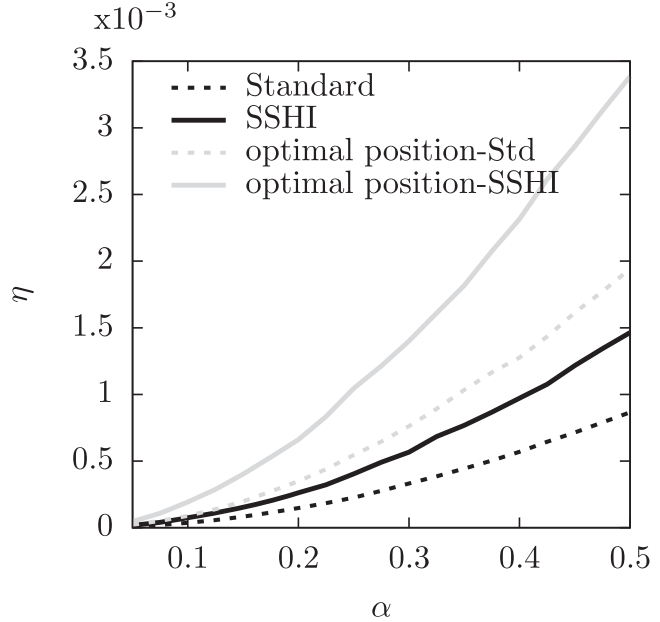

(b)

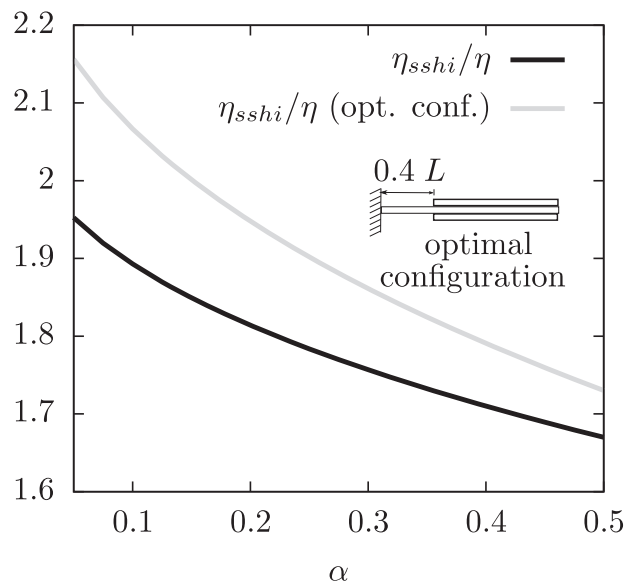

Figure 4. (a) Energy harvesting efficiency as a function of the electro-mechanical coupling $\alpha$ for standard harvesting (solid) and SSHI harvesting (dashed), in the case of a fully covered piezoelectric plate (black) and a plate with an optimally positioned piezoelectric element (gray) [14]. (b) Relative efficiency improvement introduced by the SSHI technique. $\Gamma, Q_{i}$ and $\beta$ are determined as in figure 3.

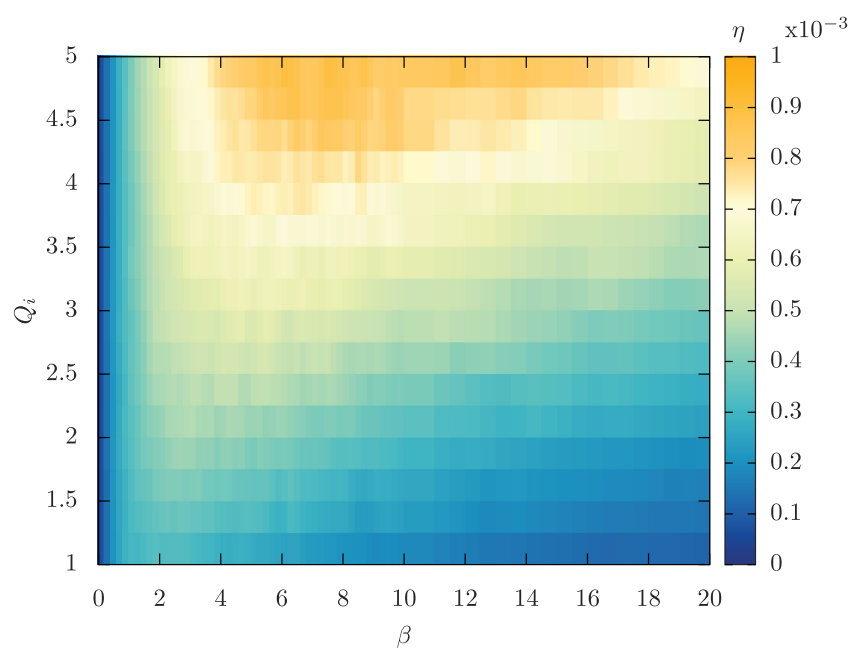

Figure 5. Energy harvesting efficiency obtained for SSHI harvesting as a function of the quality factor $Q_{i}$, with $\alpha=0.25$ and $\Gamma=180$.

\section{Conclusion}

The present work proposed an analysis of the energy harvesting efficiency of a piezoelectric flag using the SSHI technique, which has become quite popular to optimize energy harvesting from ambient vibrations. Using numerical simulations and experimental measurements, we showed that if the resistor is correctly tuned, SSHI allows for a doubling of the harvested power. A good agreement between experimental and numerical results was observed. The numerical model was further used to analyze the role of piezoelectric coupling in SSHI systems for strongly coupled systems, i.e. when the flag's dynamics is impacted by the harvesting circuit. In particular, larger coupling coefficients and quality factors have been shown to enhance the harvesting efficiency. However, due to the piezoelectric feedback coupling on the flag, the relative increase of harvested power obtained for
SSHI in comparison with the standard technique is reduced for strongly coupled systems.

Finally, using the guidelines proposed in our previous work [14], it was shown that the efficiency can again be increased by a factor 2 by optimizing the position of the harvesting electrodes along the flag's length. Hence these results show that significant improvement can be obtained if the geometries and electrical circuits of piezoelectric energy harvesters are carefully designed.

Future works should focus on the implementation of the SSHI technique on more complex systems. For example, in the case of multiple piezoelectric element systems or multiple flag energy harvesting configurations.

\section{Acknowledgments}

This work was supported by the 'Laboratoire dExcellence' LASIPS (project PIEZOFLAG) and by the French National Research Agency ANR (Grant ANR-2012-JS09-0017). MP and OD would like to thank Amalia P Pellizzi and Octave D Rouby for fruitful comments and discussions.

\section{References}

[1] Erturk A and Inman D J 2011 Piezoelectric Energy Harvesting (New York: Wiley)

[2] Chérif A, Meddad M, Belkhiat S, Richard C, Guyomar D, Eddiai A and Hajjaji A 2014 Improvement of piezoelectric transformer performances using sshi and sshi-max methods Opt. Quantum Electron. 46 117-31

[3] Bernitsas M M, Raghavan K, Ben-Simon Y and Garcia E M 2008 Vivace (vortex induced vibration aquatic clean energy): a new concept in generation of clean and renewable energy from fluid flow J. Offshore Mech. Arctic Eng. 130 041101

[4] McKinney W and DeLaurier J 1981 Wingmill: an oscillatingwing windmill J. Energy 5 109-15 
[5] Barrero-Gil A, Alonso G and Sanz-Andres A 2010 Energy harvesting from transverse galloping J. Sound Vib. 329 2873-83

[6] Tang L, Païdoussis M P and Jiang J 2009 Cantilevered flexible plates in axial flow: energy transfer and the concept of flutter-mill J. Sound Vib. 326 263-76

[7] Paidoussis M P 2004 Fluid-Structure Interactions vol 2 (London: Academic)

[8] Shelley M J and Zhang J 2011 Flapping and bending bodies interacting with fluid flows Ann. Rev. Fluid Mech. 43 449-65

[9] Allen J J and Smits A J 2001 Energy harvesting eel J. Fluids Struct. 15 629-40

[10] Doaré O and Michelin S 2011 Piezoelectric coupling in energy-harvesting fluttering flexible plates: linear stability analysis and conversion efficiency J. Fluids Struct. 27 1357-75

[11] Dunnmon J A, Stanton S C, Mann B P and Dowell E H 2011 Power extraction from aeroelastic limit cycle oscillations J. Fluids Struct. 27 1182-98

[12] Michelin S and Doaré O 2013 Energy harvesting efficiency of piezoelectric flags in axial flows J. Fluid Mech. $\mathbf{7 1 4}$ 489-504

[13] Xia Y, Michelin S and Doaré O 2015 Fluid-solid-electric lockin of energy-harvesting piezoelectric flags Phys. Rev. Appl. 3 014009

[14] Piñeirua M, Doaré O and Michelin S 2015 Influence and optimization of the electrodes position in a piezoelectric energy harvesting flag J. Sound Vib. 346 200-15

[15] Guyomar D, Badel A, Lefeuvre E and Richard C 2005 Toward energy harvesting using active materials and conversion improvement by nonlinear processing IEEE Trans.

Ultrason. Ferroelectr. Freq. Control 52 584-95

[16] Lallart M and Guyomar D 2008 An optimized self-powered switching circuit for nonlinear energy harvesting with low voltage output Smart Mater. Struct. 17035030

[17] Chen Y-Y, Vasic D, Costa F, Wu W-J and Lee C-K 2012 A self-powered switching circuit for piezoelectric energy harvesting with velocity control Eur. Phys. J. Appl. Phys. 57 30903

[18] Bisegna P, Caruso G and Maceri F 2006 Optimized electric networks for vibration damping of piezoactuated beams J. Sound Vib. 289 908-37

[19] Lighthill M J 1971 Large-amplitude elongated-body theory of fish locomotion Proc. R. Soc. B 179 125-38

[20] Candelier F, Boyer F and Leroyer A 2011 Three-dimensional extension of lighthill's large-amplitude elongated-body theory of fish locomotion J. Fluid Mech. 674 196-226

[21] Eloy C, Kofman N and Schouveiler L 2012 The origin of hysteresis in the flag instability J. Fluid Mech. 691 583-93

[22] Singh K, Michelin S and de Langre E 2012 Energy harvesting from axial fluid-elastic instabilities of a cylinder J. Fluids Struct. 30 159-72

[23] Doaré O, Mano D and Ludena J C B 2011 Effect of spanwise confinement on flag flutter: experimental measurements Phys. Fluids 23111704

[24] Xia Y, Michelin S and Doaré O 2015 Resonance-induced enhancement of the energy harvesting performance of piezoelectric flags Appl. Phys. Lett. page (to appear) (doi:10.1063/1.4939117) 


\section{QUERY FORM}

Journal: Smart Materials and Structures

Author: M Piñeirua et al

TITLE: Synchronized switch harvesting applied to piezoelectric flags

Article ID: smsaa29fc

The layout of this article has not yet been finalized. Therefore this proof may contain columns that are not fully balanced/ matched or overlapping text in inline equations; these issues will be resolved once the final corrections have been incorporated.

\section{SQ1}

Please be aware that the colour figures in this article will only appear in colour in the online version. If you require colour in the printed journal and have not previously arranged it, please contact the Production Editor now.

We have been provided funding information for this article as below. Please confirm whether this information is correct. Laboratoire d'Excellence" LASIPS: PIEZOFLAG; French National Research Agency ANR: ANR-2012-JS09-0017.

\section{Page 1}

Q1

Please specify the corresponding author and provide his/her email address.

\section{Page 4}

Q2

Table 2 was not cited in the text. Please provide the text for the citation and specify the exact location where the text should be inserted

\section{Page 8}

Q3

Please update the volume and page range in reference [24]. 